\title{
Verification of Quasi-Determinism theory against Baltic Sea Data
}

\author{
Ewa Antão \\ Institute of Meteorology and Water Management - National Research Institute, Podleśna 61, 01-673 Warsaw, \\ Poland,e-mail:ewa.antao@imgw.pl
}

\begin{abstract}
The second formulation of Boccotti's quasi-determinism theory is investigated. Deep water wave records collected in the Baltic Sea with AWAC (Nortek Acoustic Wave and Current profiler) are used in this study. The adequacy of Boccotti's theory for Baltic Sea conditions is evaluated on the basis of some simulations of wave groups with a very large maximum wave height. The large waves are chosen using an abnormality index (ratio of maximum wave height to significant wave height) with a value greater than 2 . Such values of this index are also used by different authors for the definition of freak waves. In order to obtain better results, JONSWAP formula is fitted to the measured spectra instead of using default JONSWAP parameters for the simulations. Verification of values of spectral parameters obtained from the autocovariance function against the same parameters calculated from smoothed and not smoothed spectra is presented. Much attention is given to spectral peak period value. The spectral width parameter, in QD theory known as narrow bandedness parameter, requires further investigation as the results obtained here did not allow the formulating of any functional relation with another - more often used - spectral width parameter. The low frequency of sampling of the data allowed only the most important aspects of the theory to be checked. A short Matlab function used in QD simulations is presented.
\end{abstract}

Keywords: quasi-determinism theory, deep water waves, Baltic Sea, AWAC measurements, freak waves

Submitted 15 November 2016, revised 25 November 2016, accepted 25 January 2018

\section{Introduction}

The randomness of ocean waves forces naval architects and marine engineers to use a combination of analytical and statistical wave theories in order to obtain information necessary for the design of marine structures or for the planning of marine operations. Due to the danger posed by abnormally high and steep waves, many authors have attempted to explore their nature and describe them, both statistically and analytically, as accurately as possible (Michell 1893; Haver, Andersen 2000; Pelinovsky, Karif 2000; Guedes Soares et al. 2003, 2004b; Guedes Soares, Antão 2004; Antão, Guedes Soares 2015, 2016).

In the period 1970-1979, 26 vessels and 72 lives were lost in the seas around Norway. In 13 cases survivors confirmed that their vessels capsized due to large breaking waves. These events initiated a series of studies on the steepness and asymmetry of deep water waves in Norway (Myrhaug, Kjeldsen 1984, 1986). The probability of marine structure damage connected to extreme local steepness was studied by, among others, Guedes Soares et al. (2007). Investigations on wave profiles led to the formulation of many definitions describing individual wave profiles. A good summary of these definitions can be found in Guedes Soares et al. (2004a).
Boccotti's quasi-determinism theory (QD) describes the evolution of random wave groups when either a wave with a large crest or a wave with a large height occurs at some fixed time and location. On the basis of algorithms given in Boccotti (2000), it is possible to calculate a 3D wave group profile changing in time and space in deep water and this gives better insight into some problems concerning modeling wave profiles.

The first results on maxima in Gaussian seas were obtained by Lindgren $(1970,1972)$. The complete mechanics of wave group was then derived by Boccotti (1981, 1982, 1983, 1989, 2000), who introduced the theory of quasi-determinism of the highest ocean waves. This theory was proposed in two formulations. The first one deals with the wave group mechanics when a high crest occurs in afixed time instant and point, and the latter concerns when a wave with a large height occurs (Boccotti, 2000). The study in this paper is based on the second formulation.

Boccotti (2000) presents formal proof of the consistency of QD with Stokes' theory. The main point of Stokes' theory is that the heights of waves of linear theory must be small with respect to their wavelengths and water depth, while Boccotti's theory assumes very large wave height with respect to the standard deviation of the water surface displacement in the sea state; the assumptions of both of these theories do not disagree with each other. 
A second-order contribution to both formulations of QD theory was made by Arena (2005). And Arena et al. (2008) presented several numerical applications to show how both the spectral bandwidth and the directional spreading modify the nonlinear high waves at different water depth.

In this paper, the period and height of measured maximum waves are compared to maximum waves simulated with QD theory. A more precise comparison of different profile characteristics, like in Antão et al. (2009), was not possible due to the low frequency of the sampling of the real data. Nevertheless, the simulations are more precise than in Antão et al. (2009) because theoretical spectra were fitted with JONSWAP formula (Hasselmann et al. 1973) instead of default values of parameters being used.

Besides the verification of maximum wave profile described by QD theory against Baltic Sea data, some interesting relationships between spectral and autocovariance function parameters were also investigated. These relationships allow the evaluation of the type of spectrum and its parameters without the calculation of the spectrum itself, and they could be of use for naval engineers. For instance, the narrow bandedness parameter calculated on the basis of the autocovariance function is not sensitive to high frequency noise and yields a good estimation of spectral width. Boccotti (2000) claims it is more effective than other parameters for wind dominated sea states. Another interesting point here is the probability distribution of wave heights, which he proposes. Its formula is also governed by his parameter of narrow bandedness and significant wave height. This distribution is not investigated here, however.

\section{2. $2^{\text {nd }}$ formulation of the Quasi-Determinism theory}

Wave groups, in a Gaussian sea, are characterised by profiles, in the domains of time and space, which depend on the autocovariance function of the free surface displacement. Autocovariance is the most important concept in quasi-determinism theory. Boccotti (2000) uses it to infer about the type of spectrum of the sea state. Wind dominated sea has autocovariance for which the global minimum is also the first local minimum after the origin of the coordinate system (see Figures 1, 4 and 5). Thus, together with all the wave group, the profile of the maximum wave of such a sea state can be modeled in the domains of time and space with QD theory.

Basic statistical properties of a sea state depend on the first wave of autocovariance, while the configuration of wave groups depends on the first two or three waves of it. These waves are called the core of the autocovariance.
The abscissa of the first minimum (if it is also the global minimum) of the autocovariance function $T^{*}$ serves to calculate the profile of the wave group with very large wave height (see Fig. 1).
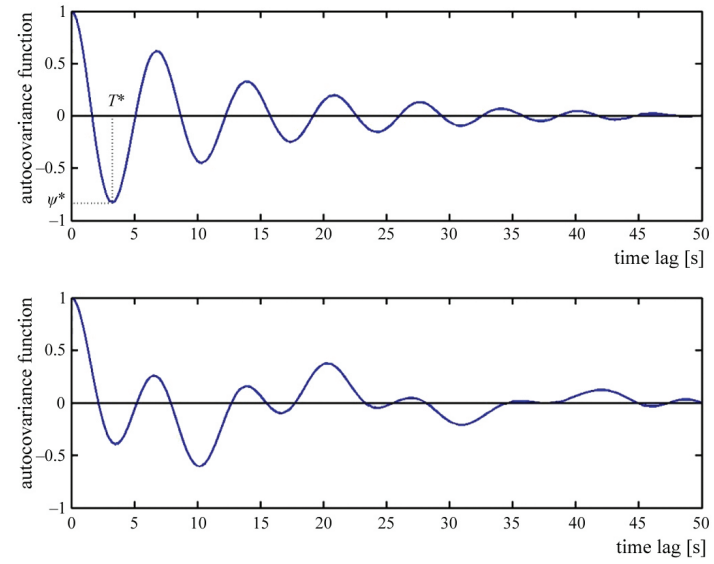

Fig. 1. Normalised autocovariance function $\psi(T) / \psi(0)$ for a sea state dominated by wind sea (upper plot) and by swell (lower plot)

Boccotti's method to calculate a continuous spectrum is based on the autocovariance expressed as a function of the variance spectrum (equation 7) but the first estimation of the spectral peak period is calculated from the autocovariance function defined with usual formula as a temporal mean:

$$
\psi(T) \equiv\langle\eta(t) \cdot \eta(t+T)\rangle
$$

then spectral peak period $T_{p}$ is approximately equal to the mean of the periods of the two highest waves of function $\psi(T)$.

Another interesting fact concerning the autocovariance of the wave record is that spectral width can be evaluated from the formula:

$$
\psi^{*}=\left|\frac{\psi\left(T^{*}\right)}{\psi(0)}\right|
$$

In the case of an infinitely narrow spectrum, the autocovariance approaches a form close to the cosine function and narrow bandedness parameter $\psi^{*}$ approaches 1 . With the growth of spectral bandwidth, the parameter $\psi^{*}$ gets smaller and decreases till zero. The narrow bandedness parameter is efficient if the absolute minimum of the autocovariance is also its first minimum after the origin of the coordinate system, as shown in Fig. 1.

The JONSWAP spectrum (Hasselmann et al. 1973) received special attention in $\mathrm{QD}$ theory. For it, the relation between the abscissa of the first minimum of the normalized autocovariance function and the spectral peak period was calculated, as well as the value, or rather narrow interval of values, of the narrow bandedness parameter, which 
is $\psi^{*} \in(0.62,0.75)$. The narrow bandedness parameter $\psi^{*}$ also appears, together with significant wave height, in the formula for probability density function for wave height in Boccotti (2000).

The second formulation of quasi-determinism theory deals with the wave group mechanics when a wave of large height occurs. More specifically, if a wave with crest-to-trough height equal to $H$, which is very large with respect to the standard deviation $\sigma$ of the free surface displacement $\left(\frac{H}{\sigma} \rightarrow \infty\right.$ or practically $\left.\frac{H}{H_{s}}>2 \Leftrightarrow \frac{H}{\sigma}>8\right)$, occurs at time $t_{0}$ at point $\left(x_{0}, y_{0}\right)$, the free surface displacement, with a probability approaching 1 , will tend to the following deterministic profile:

$$
\bar{\eta}\left(x_{0}+X, y_{0}+Y, t_{0}+T\right)=\frac{\Psi(X, Y, T)-\Psi\left(X, Y, T-T^{*}\right)}{\Psi(0,0,0)-\Psi\left(0,0, T^{*}\right)} \frac{H}{2}
$$

where $\Psi(X, Y, T)$ defines the space-time covariance:

$$
\Psi(X, Y, T) \equiv\left\langle\eta\left(x_{0}, y_{0}, t\right) \cdot \eta\left(x_{0}+X, y_{0}+Y, t+T\right)\right\rangle
$$

and $T^{*}$ is the abscissa of the absolute minimum (which is assumed to also be the first minimum) of the autocovariance $\psi(T)$ at point $\left(x_{0}, y_{0}\right)$. Equation 4 can be expressed as a function of wave spectrum:

$$
\begin{gathered}
\Psi\left(X, Y, T ; x_{0}, y_{0}\right)=A g^{2} \omega_{p}^{-4} \int_{0}^{\infty} \int_{0}^{2 \pi} S(\omega, \theta) \cos \left[2 \pi \kappa(\omega) \frac{X}{L_{p 0}}\right. \\
\left.\sin \theta+2 \pi \kappa(\omega) \frac{Y}{L_{p 0}} \cos \theta-2 \pi \omega \frac{T}{T_{p}}\right] d \theta d \omega
\end{gathered}
$$

and $S(\omega, \theta)$ is the non-dimensional directional spectrum (JONSWAP formula with directional spreading function was used by Boccotti 2000), $A$ is a parameter of JONSWAP spectrum $S(\omega, \theta), L_{p 0}$ is the wavelength corresponding to spectral peak period, and $\omega_{p}$ is the frequency of the spectral peak. The formula of JONSWAP spectrum is described by equation 6 . The non-dimensional wave number $\kappa(\omega)$ has to be calculated using the iteration method with given tolerance.

$S(\omega)=A g^{2} \omega^{-5} \exp \left[-\frac{5}{4}\left(\frac{\omega_{p}}{\omega}\right)^{4}\right] \exp \left\{\ln \chi_{1} \exp \left[-\frac{\left(\omega-\omega_{p}\right)^{2}}{2 \chi_{2}^{2} \omega_{p}^{2}}\right]\right\}(6)$

where: $\chi_{1}, \chi_{2}$ are shape parameters $\left(\chi_{1} \approx 3.3, \chi_{2} \approx 0.08\right)$; $\omega_{p}$ is the frequency of the spectral peak; $A$ is a parameter for design conditions is equal to $0.01 ; \mathrm{g}$ is the gravity acceleration $\left(9.81 \mathrm{~m} / \mathrm{s}^{2}\right)$.

Equations 3 and 4 (also 5) allow the calculation of the wave groups in any point $\left(x_{0}+X, y_{0}+Y\right)$, at any time
$\left(t_{0}+T\right)$. Thus, the theory enables the analysis of wave groups both in the time domain and in the space domain. Figure 2 shows the effect of QD simulations, though with poor resolution, just in the time domain. Nevertheless, QD simulations, on a domain dense enough, allow the obtaining of $3 \mathrm{D}$ visualisations of a wave group profile with a very high wave, and its changes in space and time, as animation.

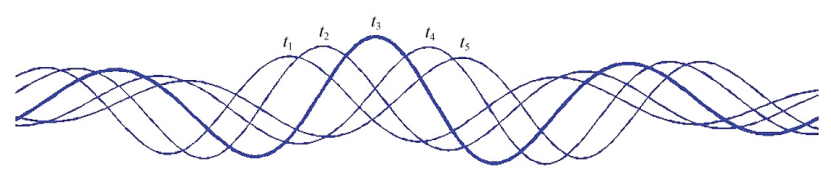

Fig. 2. Changes of wave profile while a wave travels from one (back) node of a wave group to the front node of this group; the thick line is the wave at its apex; symbols $t_{1}, \ldots, t_{5}$ over the crests of the same wave denote different time instants.

The first operation to apply equation 3 is the calculation of the autocovariance and value $T^{*}$. It can be obtained from the frequency spectrum (equation 7) and in open sea autocovariance is the same in every point of wave field:

$$
\psi(T)=\sum_{i=1}^{N} \frac{1}{2} a_{i}^{2} \cos \left(\omega_{i} T\right)
$$

where $a_{i}$ are amplitudes and $\omega_{i}$ are frequencies of harmonics of the variance spectrum.

Practically, for the simulation of a 3D wave group with a very high wave it is better to use formula for the spacetime covariance with $X=Y=0$, given in the open sea by equation 5 , from which can be obtained:

$\frac{\psi(T)}{\psi(0)}=\frac{\Psi\left(0,0, T ; x_{0}, y_{0}\right)}{\Psi\left(0,0,0 ; x_{0}, y_{0}\right)}=\frac{\int_{0}^{\infty} \int_{0}^{2 \pi} S(\omega, \theta) \cos \left(2 \pi \omega \frac{T}{T_{p}}\right) d \theta d \omega}{\int_{0}^{\infty} \int_{0}^{2 \pi} S(\omega, \theta) d \theta d \omega}$

where $\left(x_{0}, y_{0}\right)$ always denotes the position of the largest height. The right-hand side part of this equation 8 can be calculated numerically, giving $\psi(T) / \psi(0)$ as a function of $T / T_{p}$ and from here it is possible to obtain $T^{*} / T_{p}$.

The second part is calculating the water elevation $\eta$ for the wave group, in this case in deep water. Substituting in equation 3 the space-time covariance with equation 5 yields the equality:

$$
\begin{gathered}
\eta\left(\mathrm{x}_{0}+X, y_{0}+Y, t_{0}+T\right)=\frac{H}{2 D} \int_{0}^{\infty} \int_{0}^{2 \pi} S(\omega, \theta)\{\cos [A R G 1(\omega, \theta)]+ \\
-\cos [A R G 2(\omega, \theta)]\} d \theta d \omega
\end{gathered}
$$

where: 


$$
\begin{gathered}
A R G 1(\omega, \theta) \equiv 2 \pi\left[\kappa(\omega) \frac{X}{L_{p 0}} \sin \theta+\kappa(\omega) \frac{Y}{L_{p 0}} \cos \theta-\omega \frac{T}{T_{p}}\right] \\
A R G 2(\omega, \theta) \equiv 2 \pi\left[\kappa(\omega) \frac{X}{L_{p 0}} \sin \theta+\kappa(\omega) \frac{Y}{L_{p 0}} \cos \theta-\omega \frac{T-T^{*}}{T_{p}}\right] \\
D \equiv \int_{0}^{\infty} \int_{0}^{2 \pi} S(\omega, \theta)\left[1-\cos \left(2 \pi \omega \frac{T^{*}}{T_{p}}\right)\right] d \theta d \omega
\end{gathered}
$$

A formula for velocity potential can be obtained similarly; from this, particle velocities and accelerations and pressure fluctuations can be calculated, but these are not necessary for this investigation.

\section{Description of the data used}

The data was collected by AWAC - the current and directional wave profiler belonging to the Institute of Meteorology and Water Management in Gdynia. It is placed in deep waters on the sea bed of the Baltic Sea near Petrobaltic Beta platform positioned at $55^{\circ} 28^{\prime} \mathrm{N}$ and $18^{\circ} 10^{\prime} \mathrm{E}$. AWAC measures water elevation twice per hour without too many interruptions (almost continuously) throughout the year.

Some erroneous data occasionally appears. This is due to the fact that the AWAC is in the vicinity of an oil platform and vessels passing nearby can sometimes interfere with wave sensors. Also, in severe storm conditions where there are waves breaking, the AST does not detect the water surface and thus measurements are based just on pressure and at the depth of $80 \mathrm{~m}$ this will not produce usable results.

The data are time series of the length of 1024 points with water elevation sampled with a frequency of $0.75 \mathrm{~Hz}$. They are collected using the vertical beam of AWAC which is an AST beam. It measures the distance to the sea surface by use of the simple echo sounder principle. The example of one series is presented in Fig. 3. From every record, the wave spectrum is calculated and also stored. Some statistics of each sea state are calculated and in live mode are made available online.

Data collected in October, November and December 2015 is analysed here; these are 689 time series. For the purposes of this work, only series with an abnormality

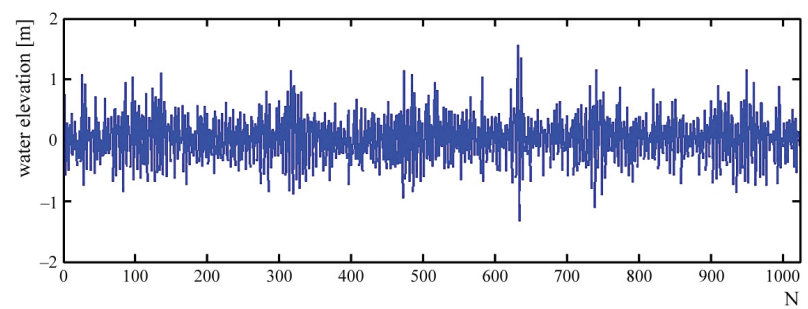

Fig. 3. Example of time series recorded by AWAC - record 24
Tab. 1. Sea state parameters of the series analysed

\begin{tabular}{|c|c|c|}
\hline No series & $\begin{array}{c}H_{s} \\
{[\mathrm{~m}]}\end{array}$ & $\begin{array}{c}T_{p} \\
{[\mathrm{~s}]}\end{array}$ \\
\hline 1 & 1.2 & 5.0 \\
\hline 6 & 1.2 & 5.3 \\
\hline 15 & 0.8 & 5.0 \\
\hline 23 & 1.2 & 5.0 \\
\hline 24 & 1.6 & 6.7 \\
\hline 28 & 1.7 & 6.3 \\
\hline 40 & 0.7 & 5.3 \\
\hline 42 & 1.1 & 6.0 \\
\hline 43 & 1.1 & 5.9 \\
\hline 44 & 1.4 & 5.0 \\
\hline 45 & 1.6 & 6.0 \\
\hline 46 & 1.6 & 6.2 \\
\hline
\end{tabular}

index (ratio of maximum wave height to significant wave height) greater than 2 were chosen - and among them, only series with a wind dominated spectrum. In total, only 12 series are left. All the series were recorded after the peak of the storm when the wind speed decreases and it is usually between 8 and $10 \mathrm{~m} / \mathrm{s}$ and when there is not strong wave-breaking turbulence (air bubbles in water prevent measurements with AWAC). All these series have an autocovariance function for which the global minimum is at the same time its first minimum - just as is shown in Fig. 4 and 5.

The autocovariance functions plotted are not very smooth. This is due to the low frequency of sampling the water elevation. Nevertheless, this should suffice for this analysis.

\section{Analysis of the results}

Based on QD theory, simulations of the profile of the wave group with the maximum wave height were prepared for the sea states described in Table 1 using the function from appendix A. For each sea state the wave group with the maximum wave was captured in 34 stages of its evolution, similar to those shown in Fig. 2. Thus, when the maximum wave becomes the highest one in the wave group - that is, some moments before it attains its apex (and vertical symmetry) - the height and period of this wave are recorded to obtain the intervals in columns 2 and 4 of Table 2 . The same is the case with the height and period of this wave after it stops being vertically symmetrical and its height starts to decrease slowly - the parameters $\left(H_{u p}\right.$ and $T_{u p}$ ) are stored till the moment the wave ceases to be the maximal one within the wave group.

It was important to capture the simulated wave and obtain some intervals for its parameters because the maxi- 

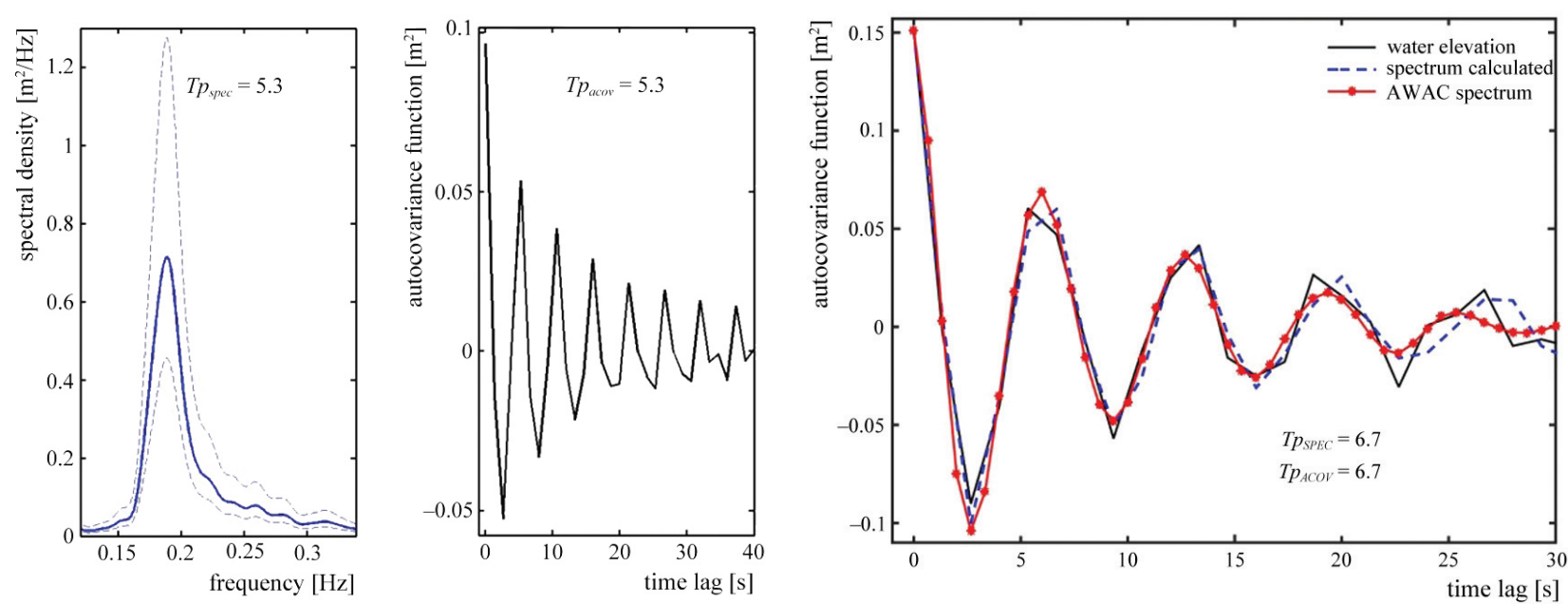

Fig. 4. Wave spectrum and autocovariance function with spectral peak values calculated from them (record 6)

Fig. 5. Autocovariance function with spectral peak values calculated from them (record 24)

mum wave measured at sea rarely is in its apex thus it is not vertically symmetrical. Neither are simulated profiles of the maximum wave just before and just after the apex moment. From this comes the necessity of using up-crossing definition for height and period even in the case of the simulated wave profiles.

The hereinbefore described way of simulating a wave group with very large waves served to prepare Table 2 for comparison of simulations with measured wave data. Looking at the table, one can see that the maximum wave period of a measured wave is very close to the interval obtained from simulations or that it falls within the limits of this interval. The distance of the real value from the interval is no more than $0.3 \mathrm{~s}$, which is equivalent to $14 \mathrm{~cm}$ of wave length for deep water. Most probably, the parameters of the real maximum wave were not measured precisely due to the low frequency of sampling.

Table 2. Parameters of maximum wave - measured and simulated with QD theory

\begin{tabular}{|c|c|c|c|c|}
\hline $\begin{array}{c}\text { No } \\
\text { series }\end{array}$ & $\begin{array}{c}H_{u p} \\
\text { simulated [m] }\end{array}$ & $\begin{array}{c}H_{u p} \\
\text { measured [m] }\end{array}$ & $\begin{array}{c}T_{u p} \\
\text { simulated [s] }\end{array}$ & $\begin{array}{c}T_{u p} \\
\text { measured [s] }\end{array}$ \\
\hline 1 & {$[1.72,2.00]$} & 1.97 & {$[4.8,4.9]$} & 5.0 \\
\hline 6 & {$[1.59,1.85]$} & 1.85 & {$[5.0,5.2]$} & 5.5 \\
\hline 15 & {$[0.86,1.02]$} & 1.00 & {$[4.7,4.9]$} & 4.9 \\
\hline 23 & {$[1.74,2.04]$} & 2.01 & {$[4.8,4.9]$} & 4.6 \\
\hline 24 & {$[2.28,2.87]$} & 2.89 & {$[5.7,6.1]$} & 6.2 \\
\hline 28 & {$[2.21,2.81]$} & 2.83 & {$[5.7,6.1]$} & 6.0 \\
\hline 40 & {$[0.77,1.09]$} & 0.97 & {$[4.4,5.0]$} & 4.8 \\
\hline 42 & {$[1.23,1.55]$} & 1.55 & {$[5.4,5.7]$} & 5.7 \\
\hline 43 & {$[1.58,1.93]$} & 1.95 & {$[5.5,5.8]$} & 5.3 \\
\hline 44 & {$[1.85,2.27]$} & 2.27 & {$[4.9,5.1]$} & 5.4 \\
\hline 45 & {$[1.50,1.86]$} & 1.87 & {$[5.4,5.8]$} & 5.9 \\
\hline 46 & {$[2.14,2.71]$} & 2.73 & {$[5.7,6.1]$} & 5.4 \\
\hline
\end{tabular}

Another probable reason explaining this small difference may be that the simulated maximum wave profile should be captured more than 34 times. Nevertheless, the result is quite satisfactory, because, contrary to maximum wave height, the maximum wave period is not given as an input for the simulations.

The usual application of QD theory allows the usage of the profile of the maximum wave at its apex only, since the further from the crest of the maximum wave the less precise the simulated profile of the wave group is. A good example of the accepted employment of QD is in Romolo and Arena (2013). To justify the construction of the intervals it was confirmed that the distance of each simulated wave crest from the apex crest is not more than one period equal to $T_{p}$ (see also Boccotti 2000, page 316-317). One expects the theory to be true for this distance, especially as the results do not say any different. Moreover, Boccotti himself uses maximum wave simulations within the distance of $T_{p}$ from the apex in order to reason about small changes in the maximum wave period.

Another question is the precision of measurements and its influence on the comparison with QD theory. The AST beam of AWAC samples the water surface with a frequency of $0.75 \mathrm{~Hz}$, which means the shortest wave that can be resolved is a $0.375 \mathrm{~Hz}$ wave (equivalent to $2.67 \mathrm{~s}$ period). Nevertheless, there exists the limit imposed by the ensonified area of the water surface, called the footprint (Pedersen, Lohrmann 2004).

The diameter of the footprint generated by the vertical AST beam of AWAC is calculated using the fact that the beam opening angle is 1.7 degrees. This and the fact that AWAC operates at $80 \mathrm{~m}$ depth yields the diameter of the AST footprint: $4.75 \mathrm{~m}$. IMGW AWAC is tilted and the tilt from vertical is 2.3 degrees, which gives a footprint of $4.76 \mathrm{~m}$. It is exactly half of the smallest wave which 
is measurable by AWAC from the depth of $80 \mathrm{~m}$. Thus, the smallest measurable wave period is $2.47 \mathrm{~s}$.

Analysing values of measured and simulated periods, one can see that they are larger than the period obtained for Nyquist frequency, which is equal to $2.67 \mathrm{~s}$. The same situation is present in the case of the period limit obtained from the diameter of the AST footprint. This means the measurements are credible enough to be used in this study.

The AST does not suffer from the attenuation effects associated with increasing depth. It measures oscillations of water elevation in the range $-15 \mathrm{~m}$ to $15 \mathrm{~m}$ with surface tracking precision of $1 \mathrm{~cm}$. This magnitude of error will not influence the conclusion from Table 2 concerning wave heights. The distances of measured waves from intervals obtained through simulations are not larger than $3 \mathrm{~cm}$, which gives $3 \mathrm{~cm} \pm 1 \mathrm{~cm}$ for waves of the height from the interval $[0.77 \mathrm{~m}, 2.89 \mathrm{~m}]$.

Boccotti (2000), as a first estimation of a spectral peak period for further calculations, suggests using the period calculated as the mean of the two highest waves of the autocovariance function. There is a peak period from the spectrum and another one from the autocovariance function presented in Table 3 (columns 2 and 5). The peak period from the spectrum in column 2 was obtained from our own calculations and not by AWAC. AWAC spectra are very smooth and usually have only one peak - for comparison see Fig. 6. This is good for automatic calculations and data presentation or in situations when one has to fit a measured spectrum with a theoretical one, like for example with JONSWAP formula, for the needs of simulations - for an assessment of the quality of these fits see Fig. 7, as well as Table 3 (columns 3 and 4).
The peak period from the autocovariance function seems to reflect the peak period value of the spectrum before smoothing. Analysing plots in Fig. 6 and comparing respective values of peak period from Table 3, columns 2, 3 and 5, for sea states 24 and 15 yields such a conclusion. One can immediately see that a higher value of spectral peak for sea state 24 , calculated from the autocovariance function, and a less smoothed spectrum differs from the AWAC peak value due to the smoother structure of the spectrum - compare Fig. 6, left plot, and Table 3, row 5 .

To confirm this conclusion see Fig. 6, right plot, and Table 3, row 3. Here both smoothed (the less smooth calculated by the author, and smoother obtained from AWAC) spectra have the same value of spectral peak, while the peak value calculated from the autocovariance function is slightly greater (frequency of the peak is smaller) and closer to the not smoothed spectrum peak value.

Another estimation of spectral peak period given by Boccotti (2000) was one with the abscissa of the first minimum (if it is also the global minimum) of the autocovariance function $T^{*}$. This was calculated and it is presented in Table 3 , column 6 . It seems very rough. The reason may be the large interval of sampling of the free water surface. Probably, in the case of the mean of two waves of the autocovariance function, the error coming from a too small frequency of sampling was more disguised and the peak value came out as more precise and closer to the real value. It is also possible that the estimation of spectral peak frequency from equality $T_{p} \approx T^{*} / 0.44$ is not as efficient as the other estimation (which is the mean of two waves of the autocovariance function - Table 3, column 5) and can only serve for a very robust approximation of the real value.

Tab. 3. Sea state parameters - calculated from slightly smoothed spectrum (column 2), AWAC spectrum (column 3), JONSWAP fit to the AWAC spectrum (column 4,7), obtained with QD (column 5, 6, 8)

\begin{tabular}{|c|c|c|c|c|c|c|c|}
\hline $\begin{array}{c}\text { No } \\
\text { series }\end{array}$ & $\begin{array}{c}T_{p} \\
\text { (calc) }\end{array}$ & $\begin{array}{c}T_{p} \\
\text { (AWAC) }\end{array}$ & $\begin{array}{c}T_{p} \\
\text { (JONSWAP fit) }\end{array}$ & $\begin{array}{c}T_{p} \\
\text { (akov) }\end{array}$ & $T_{p}=T^{*} / 0.44$ & $\begin{array}{c}\chi_{1} \\
\text { (fits) }\end{array}$ & $\psi^{*}$ \\
\hline 1 & 2 & 3 & 4 & 5 & 6 & 7 & 8 \\
\hline 1 & 5.0 & 5.0 & 5.0 & 5.3 & 6.1 & 4.1 & 0.45 \\
\hline 6 & 5.3 & 5.4 & 5.4 & 5.3 & 6.1 & 4.5 & 0.54 \\
\hline 15 & 5.0 & 5.0 & 5.0 & 5.4 & 6.1 & 2.8 & 0.35 \\
\hline 23 & 5.0 & 5.0 & 5.0 & 5.4 & 6.1 & 3.6 & 0.44 \\
\hline 24 & 6.7 & 6.3 & 6.3 & 6.7 & 6.1 & 2.5 & 0.60 \\
\hline 28 & 6.3 & 6.3 & 6.2 & 6.1 & 6.1 & 2.2 & 0.62 \\
\hline 40 & 5.3 & 5.3 & 5.1 & 5.4 & 3.0 & 1.4 & 0.30 \\
\hline 42 & 6.0 & 5.9 & 5.8 & 5.4 & 6.1 & 2.1 & 0.45 \\
\hline 43 & 5.9 & 5.9 & 5.9 & 6.0 & 6.1 & 3.2 & 0.50 \\
\hline 44 & 5.0 & 5.3 & 5.1 & 5.3 & 6.1 & 1.9 & 0.49 \\
\hline 45 & 6.0 & 5.9 & 5.9 & 6.1 & 6.1 & 2.6 & 0.53 \\
\hline 46 & 6.2 & 6.3 & 6.2 & 6.0 & 6.1 & 2.4 & 0.60 \\
\hline
\end{tabular}



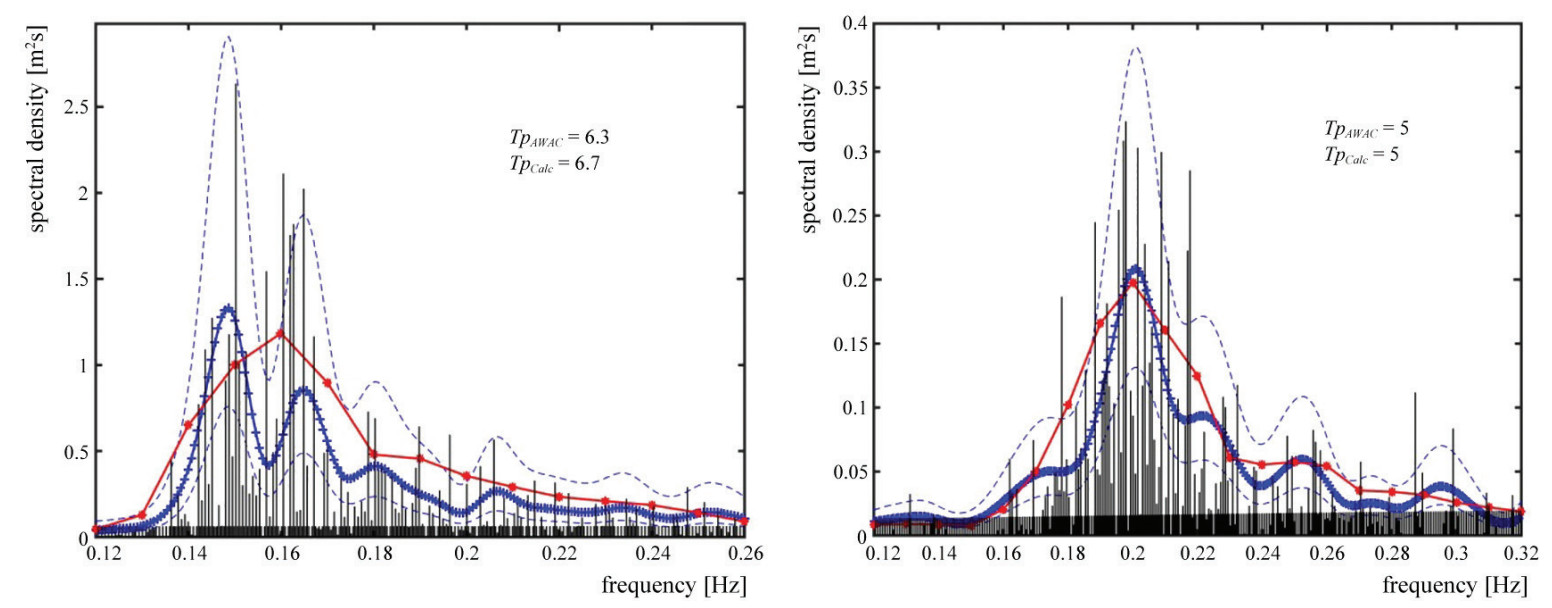

Fig. 6. Left, rec. 24 - smoother spectrum from AWAC (line with stars) results in a greater shift of peak frequency value compared to the less smooth calculated spectrum (line with crosses) and not smoothed spectrum (vertical spikes); right, rec. 15 - spectrum with smaller peak shift due to the compact structure of not smoothed spectrum.
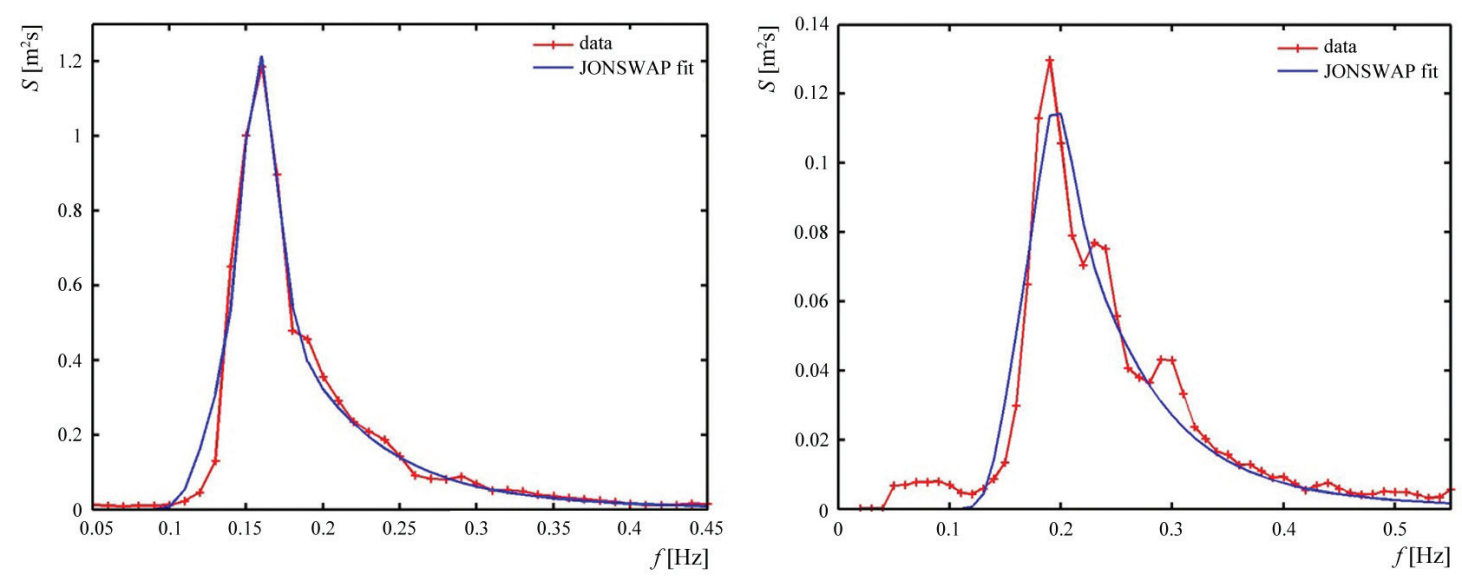

Fig. 7. Fit of JONSWAP spectrum with optimisation method to the AWAC spectrum; Left-hand side - rec. 24, better fits, right-hand side - rec. 40 , worse fits

Comparison of shapes of autocovariance function calculated from waves and from different spectra is shown in Fig. 5. There are differences in the positions of maxima and minima of the function, and for this reason it is always better to use an autocovariance function calculated directly from an instantaneous water elevation record if possible.

Fits of measured spectra (i.e., AWAC spectra) with JONSWAP formula using the optimisation method were also prepared. Some examples of such fits are presented in Fig. 7 and the values of respective spectral peaks are in Table 3, columns 3 and 4 . The fits seem to be quite satisfactory. Values of $\chi_{1}$ parameter of the JONSWAP spectrum obtained from fitting and used in QD simulations are shown in Table 3. It is visible that values from fits differ significantly from the default value of 3.3 (often used by different authors) and that this influenced QD simulations and obtained from them maximum wave parameters.

The narrow bandedness parameter $\psi^{*}$ was calculated. It is another spectral characteristic that can be evaluated quickly from the autocovariance function and without calculating the spectrum itself, as well as without concern about uncertainties coming from spectrum estimation. Boccotti's parameter neglects very high spectral frequencies. Nevertheless, they are probably not very important for engineering purposes. The values of the narrow bandedness parameter came out quite small when compared to those predicted for wind sea by Boccotti (2000) - check Table 3, last column. Values below 0.65 indicate wind waves superimposed on swell, thus a wider spectrum. Nevertheless, calculations of spectral width parameter defined by Longuet-Higgins (1983) as:

$$
v=\sqrt{\frac{m_{0} m_{2}}{m_{1}^{2}}}-1
$$

where $m_{0}, m_{1}$, and $m_{2}$ are spectral moments, gave values of $n$ belonging to the interval $[0.31,0.38]$ for investigated sea states. This means that these series are of a very narrow spectral band. It is difficult to interpret the obtained result. The small frequency of the data sampling could partly explain this problem. 


\section{Conclusions}

Boccotti's theory of quasi-determinism (Boccotti 2000) was verified against Baltic Sea deep water wave measurements using simulations of profiles of wave groups with the maximum wave of a sea state. The data was collected with AWAC. The fairly large sampling interval made the maximum wave profile not precise enough for investigation on different individual wave steepness parameters. Nevertheless, basic parameters, such as wave period and height, were investigated. The values of maximum wave period simulated and measured seem to be close enough to allow the statement that the theory gives a satisfying approximation of measurements, taking into account the low sampling frequency of the measured data.

It was confirmed that QD theory is an excellent tool for the estimation of a basic sea state parameter, namely spectral peak period, directly from the autocovariance function. This allows the calculation and smoothing of the spectrum, which contribute to errors, to be avoided.

Boccotti's spectral narrow bandedness parameter was calculated and compared with the spectral width parameter from Longuet-Higgins. Establishing any functional relation between both parameters was not successful. More investigation is necessary on this subject because, due to the low frequency of sampling and autocovariance function not being smooth enough, it was difficult to interpret the obtained result.

In order to encourage the testing of QD theory against different data, and also to avoid lengthy explanations concerning calculations, the main function used to simulate the theory in this investigation is given in appendix A.

\section{APPENDIX A}

Matlab function to calculate the wave group profile with the maximum wave height in a series.

$\mathrm{X}=0 ; \mathrm{Y}=[-0.5: 0.03: 0.5] ' * \mathrm{Lp} ; \mathrm{T}=[4: 0.1: 4] .{ }^{*} \mathrm{Tp}$;

function $[\mathrm{x}, \mathrm{y}, \mathrm{t}, \mathrm{eta}]=$ waterElev $(\mathrm{X}, \mathrm{Y}, \mathrm{T}$, theta, $\mathrm{w}, \mathrm{k}, \mathrm{Hmax}, \mathrm{Tp}$, domin_theta,Ts,chi1)

$\%$ waterElev calculates water elevation in points given by vectors X,Y,T on the basis of Quasideterminism Theory $\%$

$\%$ CALL: $[\mathrm{x}, \mathrm{y}, \mathrm{t}, \mathrm{eta}]=$ waterElevForAnim $(\mathrm{X}, \mathrm{Y}, \mathrm{T}$, theta, $\mathrm{w}, \mathrm{k}$, Hmax,Tp,domin_theta,Ts,chi1);

$\%$

$\%$ input:

$\% \mathrm{X}, \mathrm{Y}, \mathrm{T}-$ are vectors, which are coordinates in space and time of a point for which we want to calculate the water elevation

$\%$ theta - is a vector of angles
$\% \mathrm{w}-$ is a vector of dimensionless angular frequency

$\% \mathrm{k}-$ is a vector of dimensionless wave number corre-

sponding to $\mathrm{w}$ its length $=$ length $(\mathrm{w})$

$\%$ Hmax - maximum wave height in the time series

$\% \mathrm{Tp}-$ spectral peak period

$\%$ domin_theta - spectral peak period

$\%$ Ts - the abscissa of the absolute minimum of autocovariance $\mathrm{fi}(\mathrm{T})$

$\%$ chil - parameter of fitted JONSWAP spectrum

$\%$

$\%$ output:

$\%$ eta - water elevation in the point $\mathrm{X}, \mathrm{Y}$ and time $\mathrm{T}$

$\% \mathrm{x}, \mathrm{y}, \mathrm{t}-$ are vectors, which are coordinates in space and time for water elevation values in eta

$\%$

$\%$ Example:

$\%[\mathrm{x}, \mathrm{y}, \mathrm{t}, \mathrm{eta}]=$ waterElevForAnim $(\mathrm{X}, \mathrm{Y}, \mathrm{T}$, theta, $\mathrm{w}, \mathrm{k}, \mathrm{Hmax}$, Tp,domin_theta,[],chi1);

$\%$ bibl. Boccotti p. 312

$\%$

if nargin $<10 \mid$ isempty(Ts)

Ts $=0.44 * \mathrm{Tp} ; \%$ better use real value

end

domin theta $=$ domin_theta*pi/180; \% degrees to $\mathrm{rad}$

d_w $=\mathrm{w}(2)-\mathrm{w}(1)$;

d theta $=$ theta(2)-theta(1); $\%$

$\% 5 \mathrm{D}$ arrays $\mathrm{X}, \mathrm{Y}, \mathrm{T}$, theta, $\mathrm{W}$ and $\mathrm{k}$ for the calculation of eta $\mathrm{X} 1=\mathrm{X} ; \%$ (1D)

$\mathrm{Y} 1=\mathrm{Y} ; \%(1 \mathrm{D})$

$\mathrm{T} 1=\mathrm{T} ; \%(1 \mathrm{D})$

theta $1=$ theta; $\%(1 \mathrm{D})$

$[\mathrm{X}, \mathrm{Y}, \mathrm{T}$, theta, $\mathrm{w}]=\operatorname{ndgrid}(\mathrm{X} 1, \mathrm{Y} 1, \mathrm{~T} 1$, theta1, $\mathrm{w}) ; \quad \%$

$\mathrm{X}, \mathrm{Y}, \mathrm{T}$, theta,k arrays (5D)

$[\mathrm{X}, \mathrm{Y}, \mathrm{T}$, theta, $\mathrm{k}]=\operatorname{ndgrid}(\mathrm{X} 1, \mathrm{Y} 1, \mathrm{~T} 1$, theta $1, \mathrm{k}) ; \% \mathrm{w}$ array (5D)

$\%$

$\%$ Nondimensional JONSWAP Spectrum

$\%$ chi $1=3.3 ; \%$ better use real value

chi $2=0.08$

aux $=\exp \left(-(\mathrm{w}-1) \cdot{ }^{\wedge} 2 \cdot /\left(2 \cdot{ }^{*} \operatorname{chi} 2 \cdot{ }^{\wedge} 2\right)\right)$;

$\mathrm{SD} 1=\mathrm{w} .{ }^{\wedge}-5 \cdot{ }^{*} \exp \left(-5 . / 4{ }^{*} \mathrm{w} .{ }^{\wedge}-4\right) \cdot{ }^{*} \exp \left(\log (\mathrm{chi} 1) \cdot{ }^{*} \mathrm{aux}\right)$;

$\%$

\% Directional Spreading Function

$\mathrm{n} \_\mathrm{p}=20$;

$\%$ calculate $\mathrm{n}(\mathrm{w})$

$\mathrm{n}=\mathrm{n} \_\mathrm{p} .{ }^{*} \mathrm{w} . \wedge 5$;

positions $=$ find $(\mathrm{w}>1)$;

$\mathrm{n}($ positions $)=\mathrm{n} \_\mathrm{p} .{ }^{*} \mathrm{w}($ positions $){ }^{\wedge}-2.5$; 
$\%$ calculate normalisation factor

$\operatorname{aux}=\left((\cos (\right.$ theta. $\left./ 2)) \cdot{ }^{\wedge} 2\right) \cdot \wedge n$;

aux $=\operatorname{sum}($ aux, 4$) . * d_{\text {_theta; }}$

normalisationFactor $=1$. aux;

$\%$ normalisationFactor 5 dimensional array

$[\mathrm{X}, \mathrm{Y}, \mathrm{T}$, theta,normalisationFactor $]=\ldots$

$\operatorname{ndgrid}(\mathrm{X} 1, \mathrm{Y} 1, \mathrm{~T} 1, \ldots$ theta $1, \ldots$

squeeze(normalisationFactor $(1,1,1,1,:))$ );

$\%$ calculate directional spreading function

aux $=\cos (($ theta-domin_theta $) . / 2)$;

$\mathrm{D}=$ normalisationFactor. $*(\operatorname{aux} . \wedge 2) . \wedge \mathrm{n}$; $\%$

$\%$ Directional Spectrum

S_dir $=$ SD $1 . * D$

$\%$

$\%$ Water elevation (wave group with a large wave)

$\mathrm{Lp} 0=1.56^{*} \mathrm{Tp}^{\wedge} 2$

ARG1 = 2.*pi. ${ }^{*}\left(\mathrm{k} .{ }^{*} \mathrm{X} . / \mathrm{Lp} 0 .{ }^{*} \sin (\right.$ theta $)+\mathrm{k} .{ }^{*} \mathrm{Y} . /$

Lp0.*cos(theta)-w.*T./Tp);

$\mathrm{ARG} 2=\mathrm{ARG} 1+2 .{ }^{*} \mathrm{pi} .{ }^{*} \mathrm{w} .{ }^{*} \mathrm{Ts} . / \mathrm{Tp}$;

D0 $=\operatorname{sum}(\operatorname{sum}($ S_dir.*(1-cos(2.*pi.*w.*Ts./Tp $)), 5), 4) .{ }^{*}$ d theta.*d_w;

$\operatorname{aux}=\operatorname{sum}\left(\operatorname{sum}\left(\mathrm{S} \_\right.\right.$dir.* $\left.\left.*(\cos (\mathrm{ARG} 1)-\cos (\mathrm{ARG} 2)), 5\right), 4\right)$;

eta $=$ Hmax. $/ 2 . *$ aux. $*$ d_theta.*d_w./D0;

$\mathrm{X}=\mathrm{X}(:,:, 1,1,1)$;

$\mathrm{y}=\mathrm{Y}(:,:, 1,1,1)$;

$\mathrm{t}=\mathrm{T} 1$;

figure

eta $=$ squeeze $($ eta $)$

plot(t,eta,'+-');

$\%$

Bibliography

Antão E., Arena F., Guedes Soares C., Romolo A., 2009, Steepness of high ocean waves in Quasi-Determinism theory, ASME Proceedings, Paper No. OMAE2009-79876, $597-$ 604, DOI: 10.1115/OMAE2009-79876

Antão E., Guedes Soares C., 2015, Joint distributions of wave steepness in narrow band sea states, Ocean Engineering, 101, 201-210, DOI: 10.1016/j.oceaneng.2015.04.007

Antão E., Guedes Soares C., 2016, Approximation of the joint probability density of wave steepness and height with a bivariate gamma distribution, Ocean Engineering, 126, 402-410, DOI: 10.1016/j.oceaneng.2016.09.015

Arena F., 2005, On non-linear very large sea wave groups, Ocean Engineering, 32 (12), 1311-1331, DOI: 10.1016/j.oceaneng.2004.12.002

Arena F., Ascanelli A., Nava V., Pavone D., Romolo A., 2008, Three-dimensional nonlinear random wave groups in inter- mediate water depth, Coastal Engineering, 55 (12), 10521061, DOI: 10.1016/j.coastaleng.2008.04.002

Boccotti P., 1981, On the highest waves in a stationary Gaussian process, Atti della Accademia Ligure di Scienze e Lettere, $38,271-302$

Boccotti P., 1982, On ocean waves with high crests, Meccanica, 17 (1), 16-19, DOI: 10.1007/BF02156003

Boccotti P., 1983, Some new results on statistical properties of wind waves, Applied Ocean Research, 5 (3), 134-140, DOI: 10.1016/0141-1187(83)90067-6

Boccotti P., 1989, On mechanics of irregular gravity waves, Atti della Accademia Nazionale dei Lincei. Memorie, 15 (19), 110-170

Boccotti P., 2000, Wave mechanics for ocean engineering, vol. 64, Elsevier Science, Oxford, 520 p.

Guedes Soares C., Antão E., 2004, Comparison of the characteristics of abnormal waves on the North Sea and Gulf of Mexico, [in:] Rogue Waves 2004: Proceedings of a Workshop Organized by Ifremer and Held in Brest, France, 20-22 October, M. Olagnon, M. Prevosto (eds.), Editions Quae

Guedes Soares C., Cherneva Z., Antão E., 2003, Characteristics of abnormal waves in North Sea storm sea states, Applied Ocean Research, 25 (6), 337-344, DOI: 10.1016/j. apor.2004.02.005

Guedes Soares C., Cherneva Z., Antão E., 2004a, Steepness and asymmetry of the largest waves in storm sea states, Ocean Engineering, 31 (8-9), 1147-1167, DOI: 10.1016/j.oceaneng.2003.10.014

Guedes Soares C., Cherneva Z., Antão E., 2004b, Abnormal waves during Hurricane Camille, Journal of Geophysical Research, 109 (C8), DOI: 10.1029/2003JC002244

Guedes Soares C., Pascoal R., Antão E., Voogt A., Buchner B., 2007, An approach to calculate the probability of wave impact on an FPSO bow, Journal of Offshore Mechanics and Arctic Engineering, 129 (2), 73-80, DOI: 10.1115/1.2426983

Hasselmann K., Barnett T.P., Bouws E., Carlson H., Cartwright D.E., Enke K., Ewing J.A., Gienapp H., Hasselmann D.E., Kruseman P., Meerburgh A., Muller P., Olbers D.J., Richter K., Sell W., Walden H., 1973, Measurement of wind-wave growth and swell decay during the Joint North Sea Wave Project (JONSWAP), Deutsche Hydrographische Zeitschrift. Ergänzungsheft. Reihe A, No 12, Hamburg, 95 p.

Haver S., Andersen O.J., 2000, Freak waves - myth or reality?, Technical Report OOS97*15547, STATOIL, Trondheim, Norway

Lindgren G., 1970, Some properties of a normal process near a local maximum, The Annals of Mathematical Statistics, 41 (6), 1870-1883

Lindgren G., 1972, Local maxima of Gaussian fields, Arkiv för Matematik, 10 (1-2), 195-218, DOI: 10.1007/BF02384809 
Longuet-Higgins M.S., 1983, On the joint distribution of wave periods and amplitudes in a random wave field, Proceedings of the Royal Society A. Mathematical, Physical and Engineering Sciences, 389, 241-258, DOI: 10.1098/rspa.1983.0107

Michell J.H., 1893, On the highest wave in water, The London, Edinburgh, and Dublin Philosophical Magazine and Journal of Science, 36 (222), 430-437, DOI: $10.1080 / 14786449308620499$

Myrhaug D., Kjeldsen S.P., 1984, Parametric modelling of joint probability density distributions for steepness and asymmetry in deep water waves, Applied Ocean Research, 6 (4), 207-220, DOI: 10.1016/0141-1187(84)90059-2

Myrhaug D., Kjeldsen S.P., 1986, Steepness and asymmetry of extreme waves and the highest waves in deep water, Ocean Engineering, 13 (6), 549-568, DOI: 10.1016/00298018(86)90039-9
Pedersen T., Lohrmann A., 2004, Possibilities and limitations of Acoustic Surface Tracking, Ocean '04 - MTS/IEEE TechnoOcean '04: Bridges across the Oceans - Conference Proceedings, 3, 1428-1434, DOI: 10.1109/OCEANS.2004.1406331

Pelinovsky E., Karif C., 2000, Nonlinear wave focusing a mechanism of the freak wave generation in the ocean, [in:] Rogue Waves 2000: Proceedings of a Workshop Organized by Ifremer and Held in Brest, France, 29-30 November, M. Olagnon, G.A. Athanassoulis (eds.), Editions Quae

Romolo A., Arena F., 2013, Three-dimensional non-linear standing wave groups: Formal derivation and experimental verification, International Journal of Non-Linear Mechanics, 57, 220-239, DOI: 10.1016/j.ijnonlinmec.2013.08.005 